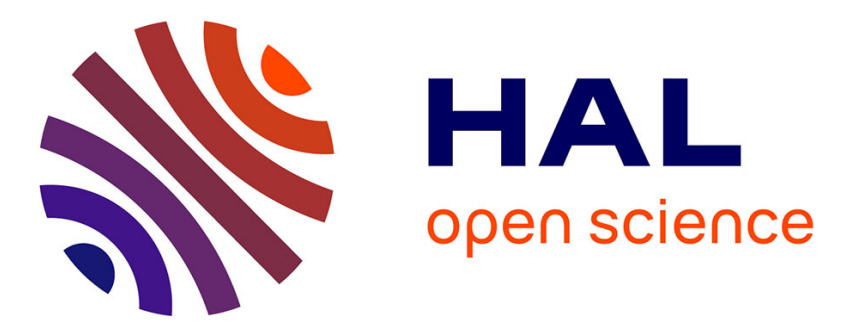

\title{
All-optical measurement of residual chromatic dispersion and OSNR using self-phase modulation in optical fiber
}

Clément Courvoisier, Julien Fatome, Christophe Finot

\section{To cite this version:}

Clément Courvoisier, Julien Fatome, Christophe Finot. All-optical measurement of residual chromatic dispersion and OSNR using self-phase modulation in optical fiber. 13th International Conference on Transparent Optical Network, Jun 2011, Stockholm, Sweden. pp.We.D1.4. hal-00586528

\section{HAL Id: hal-00586528 \\ https://hal.science/hal-00586528}

Submitted on 30 Jun 2011

HAL is a multi-disciplinary open access archive for the deposit and dissemination of scientific research documents, whether they are published or not. The documents may come from teaching and research institutions in France or abroad, or from public or private research centers.
L'archive ouverte pluridisciplinaire $\mathbf{H A L}$, est destinée au dépôt et à la diffusion de documents scientifiques de niveau recherche, publiés ou non, émanant des établissements d'enseignement et de recherche français ou étrangers, des laboratoires publics ou privés. 


\title{
All-optical measurement of residual chromatic dispersion and OSNR using self-phase modulation in optical fiber
}

\author{
Clément Courvoisier, Julien Fatome and Christophe Finot \\ Laboratoire Interdisciplinaire Carnot de Bourgogne, UMR 5209, 9 Av. A. Savary, Dijon, France \\ Christophe.Finot@u-bourgogne.fr
}

\section{ABSTRACT}

We describe a new all-fibered device providing high accuracy all-optical measurements of both optical signal to noise ratio and residual chromatic dispersion. Taking advantage of the self-phase modulation driven spectral evolution of pulses propagating in a highly non-linear medium, we have numerically and experimentally validated the benefits of using an anomalous dispersive fiber combined with a central spectral filtering.

Keywords: Optical performance monitoring, Nonlinear optics, Optical fiber, Optical Telecommunication.

\section{INTRODUCTION}

Following the development of high-speed optical networks and associated increasing bit-rates and complexity, it has become of a great importance to benefit from efficient devices able to accurately measure the various impairments degrading the data stream during its propagation in optical fiber. Consequently, all-optical solutions enabling a direct performance monitoring are a growing field of investigation to detect and compensate for the performance fluctuations of these systems [1].

Among the large panel of techniques that have already been studied, several methods based on nonlinear devices have so far demonstrated their potential to monitor the level of residual chromatic dispersion (RCD) as well as optical signal to noise ratio (OSNR). We can underline several reported techniques based on semiconductors optical amplifiers [2] or nonlinear effects occurring in optical fibers, including stimulated Brillouin scattering [3] and Kerr nonlinearity. In the latter case, several configurations have been experimentally tested such as : Kerr nonlinearity in a nonlinear optical loop mirror [4], parametric processes [5], cross-phase modulation [6] or self-phase modulation (SPM) taking place within normally dispersive fibers associated with an offset spectral filtering [7].

In the present contribution, we propose and experimentally demonstrate a new configuration enabling both monitoring of the RCD and OSNR of a return-to-zero telecommunication signal. Compared to ref. [7], our set-up benefits from the SPM effect occurring in an anomalous dispersion fiber and requires an output centered optical bandpass filtering (OBPF). We first describe the principle of operation as well as the experimental set-up of our device. In the following sections, experimental and numerical results outline a high sensitivity relative to the two impairments.

\section{EXPERIMENTAL SETUP AND PRINCIPLE OF OPERATION}

Our experimental setup is illustrated in Fig 1a. A 10-GHz modelocked fiber laser (MLL) delivers ultrashort pulses with a full width at half-maximum (FWHM) of $2.4 \mathrm{ps}$ and with a central wavelength of $1550 \mathrm{~nm}$. A dual intensity/phase programmable optical filter is then inserted in order to emulate the RCD by providing a parabolic phase profile to the incoming pulses. In parallel, the initial signal is also degraded by means of its combination with a spectrally filtered amplified spontaneous emission source (ASE). By tuning the level of spectral attenuation of the dispersion emulator, a direct control of the OSNR is thus achieved.
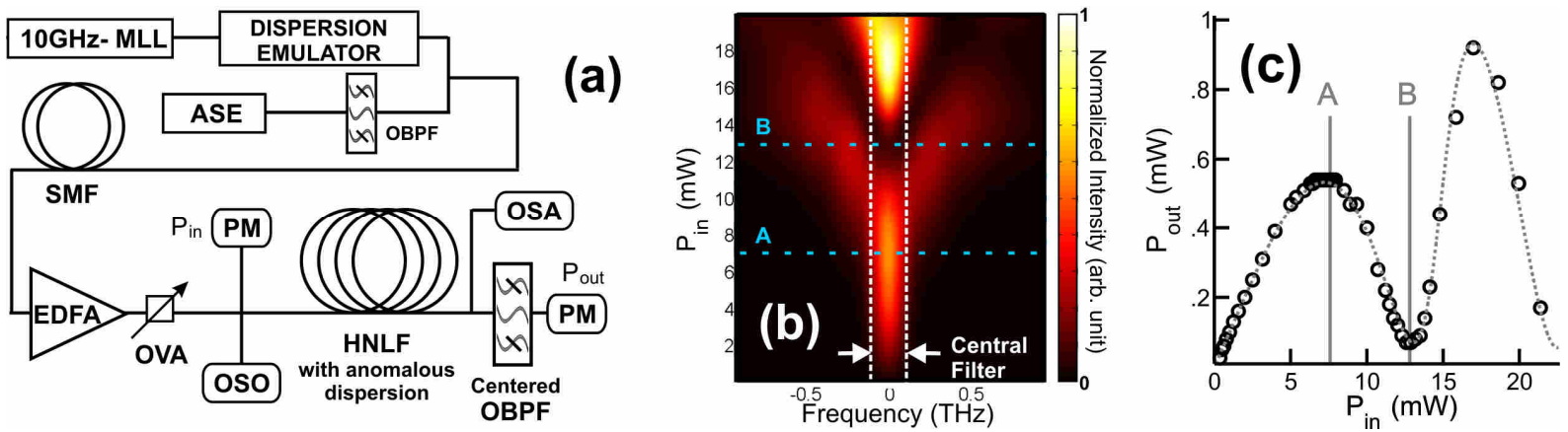

Figure 1. (a) Experimental setup. PM : powermeter. OVA : optical variable attenuator $\quad$ (b) Evolution of the experimental optical spectrum monitored after the HNLF as a function of input average power. Dashed white line illustrates the FWHM of the OBPF. (circles) are compared with numerical simulations (dashed grey line). 
The core of our all-fibered device relies on a 920-m long highly nonlinear fiber (HNLF, fiber from ofs fitel) characterized by a Kerr coefficient $\gamma$ of $10 \mathrm{~W}^{-1} \cdot \mathrm{km}^{-1}$ and an anomalous dispersion $\beta_{2}$ of $0.7 \mathrm{ps} / \mathrm{km} / \mathrm{nm}$. The linear losses $(0.5 \mathrm{~dB} / \mathrm{km})$ and dispersion slope $\left(0.01 \mathrm{ps} / \mathrm{km} / \mathrm{nm}^{2}\right)$ are both reduced so their global impact can be neglected. In order to achieve an adequate working power (WP) and thus to observe the expected nonlinear behavior, an erbium-doped fiber amplifier (EDFA) combined with a variable attenuator (so as to finely tune the WP) is inserted before injection into the HNLF. An additional segment of standard single mode fiber SMF (31 $\mathrm{m})$ is also used and its effect will discussed in $\S 3$.

During their propagation in the HNLF, optical pulses experience a significant spectral expansion provided by the SPM effect, as highlighted in the experimental measurements depicted in Fig. 1b. Note that this spectral broadening is also characterized by an atypical depletion of the central part of the spectrum for input average powers near $13 \mathrm{~mW}$ (point B). For higher power levels, solitonic compression leads naturally to general enrichment of the spectrum.

At the fiber output, a 100-GHz Gaussian-shape OBPF centered on the signal wavelength (see dashed white line, Fig. 1b) carves into the resulting expanded spectrum.

The fundamental feature of our system is the shape of its intensity transfer function (TF) that non-linearly maps the output average power obtained after filtering as a function of the input average power. Indeed, according to the choice of fiber parameters, three different behaviors can be obtained : a monotonously increasing TF, a curve exhibiting a saturated plateau area or a raise and fall evolution [8]. At the opposite of classical applications that usually target an all-optical ultrafast power-limiter and where a plateau zone is suitable $[9,10]$, we voluntarily implement here a strong rise and fall evolution. Indeed, such a TF characteristic enables an enhanced sensitivity towards the initial pulse stream fluctuations and has recently been experimentally exploited in the context of an ultrafast all-optical amplitude jitter magnifier [11] and in a bit error monitoring scheme [12].

The experimental data for the TF of our device are plotted in Fig. 1c (open circles) and are in very good agreement with the results obtained from numerical simulations of the standard nonlinear Schrödinger equation (dash grey line), with $\psi, z$ and $t$ being the electrical field, the longitudinal and temporal coordinates respectively :

$$
i \frac{\partial \psi}{\partial z}=\frac{\beta_{2}}{2} \frac{\partial^{2} \psi}{\partial t^{2}}-\gamma|\psi|^{2} \psi
$$

It is important to notice that the dramatic depletion of the central part in the spectrum observed for input powers close to $13 \mathrm{~mW}$ (point $\mathrm{B}$ ) is responsible for the minimum of output power observed on the TF.

\section{CHROMATIC DISPERSION MONITORING}

In this section, we focus on the capability of our device to efficiently highlight the consequences of the presence of RCD. We consider in our discussion an input signal only affected by RCD and not by other sources of impairments such as polarization mode dispersion or cross/self phase modulation. As a result, the parabolic spectral phase imposed by the initial dispersion emulator directly impacts the temporal width of the pulses entering into the HNLF as can be seen in Fig. 2a, where the temporal duration of pulses has been measured by an optical sampling oscilloscope, OSO (see for example insets of Fig. 2a). Note that the fixed 31-m long segment of SMF imposes an additional chirp on the incoming pulses, so that the minimum temporal duration was not obtained for a RCD equals to zero.

For a fixed average power, the amount of RCD directly acts on the input peak power of the pulses so that the resulting spectral broadening would be severely affected. Indeed, the SPM occurring into the HNLF basically depends on the temporal gradient of the intensity profile, in other words, on both peak-power and temporal duration. Fig. 2b illustrates such a dependence for an input average power of $7.25 \mathrm{~mW}$ (ie, working point corresponding to the point A on Fig. 1c). This sensitivity is also readily apparent on the TF : Fig. 2c1 summarizes the TF simulated for various amounts of RCD and underlines a continuous expansion of the TF towards higher average input powers. The experimental results, plotted in Fig. 2c2 are once again in good agreement with these numerical predictions and emphasize the high sensitivity of our device towards the RCD. Consequently, if for a practical use of our device we operate at a fixed input average power, we can make out that the output average power will be strongly affected by the level of RCD (Fig. 2d). To illustrate this point, we have considered the evolution of the output average power as a function of RCD for two different initial WP, corresponding to the local extrema of the TF (with no RCD): maximum A (black crosses) and minimum B (grey crosses) as defined in Fig. 1c.

In the case of a WP A $(7.25 \mathrm{~mW}$, black crosses), the evolution of the output power as a function of level of $\mathrm{RCD}$ is monotonously increasing in the range of -1 to $2 \mathrm{ps} / \mathrm{nm}$. Moreover, for this span, one can not only get access to the absolute value of the RCD, but also to its sign, which was not obvious with previously demonstrated techniques and which could be considered as crucial to ensure a convenient feedback from a tunable dispersion compensator. This extended range of unambiguous measurement is closely linked to the addition of the SMF pre-chirp: without such a fiber, the measurable RCD would have been limited to -0.3 to 2 ps/nm, as shown by numerical results plotted with a dashed black curve in Fig. 2 d. 
If we now operate at the WP B (12.9 mW, grey crosses), the system exhibits a higher sensitivity to the level of RCD. However, this is at the cost of the information regarding the sign of the RCD. In this context, combining the measurements at working powers A and B could be beneficial to measure accurately low RCD levels: WP A providing the sign of the RCD as well as a rough estimation, while the absolute value can be then more finely determined using WP B.
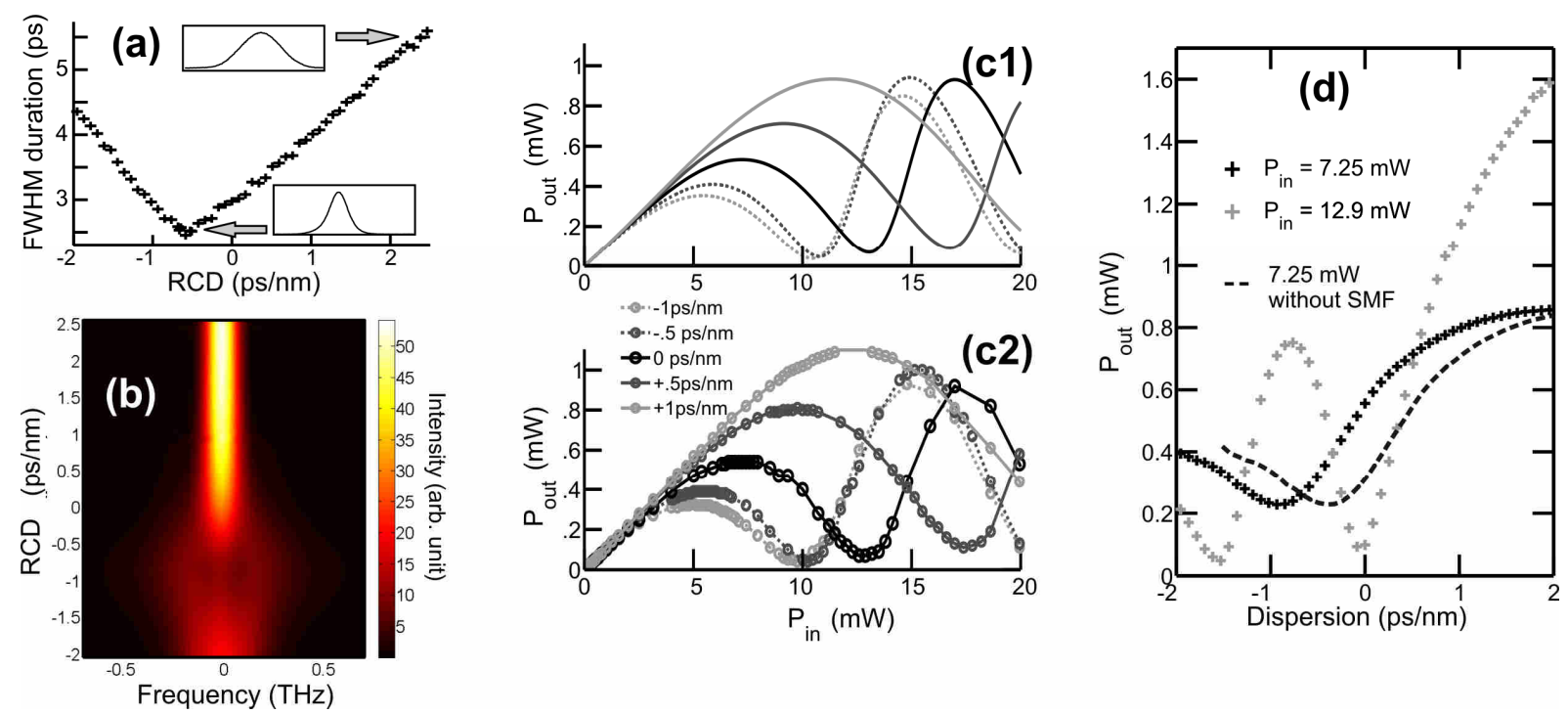

Fig. 2: (a) FWHM temporal duration of the pulse entering the HNLF according to the RCD induced by the dispersion emulator. Insets : temporal intensity profiles recorded over a time span of 15 ps. $\quad(b)$ Experimental demonstration of the influence of the RCD on the resulting spectrum recorded at the output of the HNLF and for a working average power of $7.25 \mathrm{~mW}$.

(c) TF for various amounts of RCD. Numerical simulations (c1) are compared with experimental results (c2). (d) Influence of the RCD on the output power for two WPs : initial average power of $7.25 \mathrm{~mW}$ (black crosses) and $12.9 \mathrm{~mW}$ (grey crosses). The results without the SMF (numerical simulations) are plotted using a dashed black line.

\section{IMPACT OF OSNR}

In this last section, we investigate the impact of the in-band optical signal to noise ratio (OSNR) on the output average power. In this series of experiments and for a fixed RCD, the OSNR is simply tuned by varying the level of signal mixed with the additional ASE, see Fig. 1a.

Experimental results are summarized in Fig. 3a and show that for a degraded OSNR, a higher input power is required to observe the same TF. This can be physically understood given the fact that, for a fixed temporal pulse profile, the SPM-driven spectral broadening is highly sensitive to the peak-power, thus directly related to the energy contained into the pulse. Consequently, a decreased OSNR will require a higher average power to compensate for the energy contained in the noise and therefore to maintain the same pulse energy. We can make out that by operating at WP B, the output power is directly correlated to the OSNR. As shown by experimental results depicted in Fig. 3b, the output power varies continuously from 0.1 to $0.55 \mathrm{~mW}$ for an OSNR ranging from -32 to $-15 \mathrm{~dB}$ with a remarkably linear part over $10 \mathrm{~dB}$. Hence, the direct measurement of the output power for a fixed WP can enable us to easily monitor the OSNR of the incoming signal.
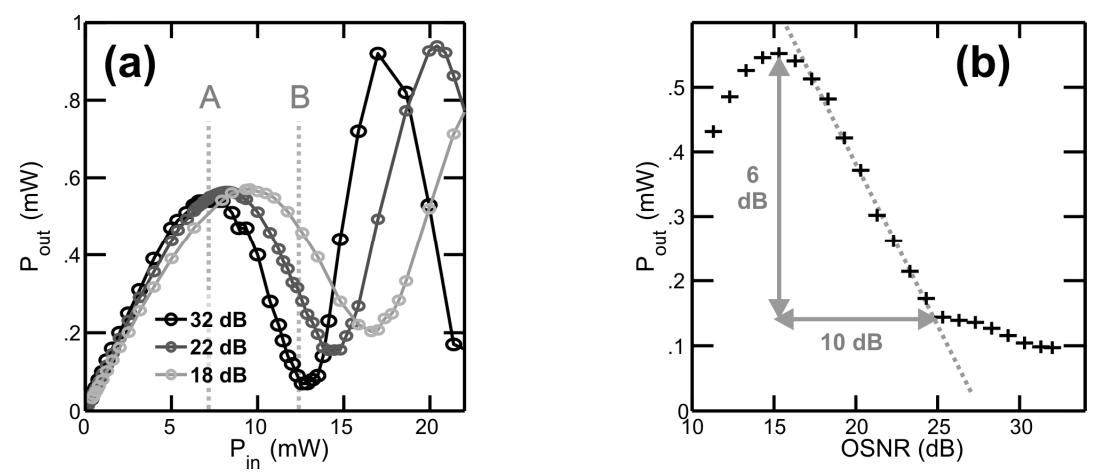

Fig. 3: (a) Influence of the OSNR on the TF. (b) Output average power as a function of the input signal OSNR for a working power of $12.9 \mathrm{~mW}$. 
Quite interestingly, if one can record the TF in presence of both RCD and OSNR, one should be able to discriminate the consequences of each effect. More precisely, the easy-to-implement following strategy that focuses on point A (defined as the first local maximum of the TF, see Fig. 1b and 1c) could be used. From Fig. 3a, it can be noticed that the output power for this point is nearly not affected by the OSNR. On the contrary, at this point, the RCD has a strong impact on the output power (Fig. 2c). Consequently, the value of the RCD could be extracted from the output power recorded at this maximum of the TF. Once the RCD value is known (and provided calibration of the influence of the OSNR), we can then use the value of the working power required for point $\mathrm{A}$, this WP being highly affected by the OSNR (see Fig. 3a).

\section{CONCLUSIONS}

We have proposed and experimentally demonstrated a new practical all-optical architecture that provides direct measurement of both optical signal to noise ratio and level of residual chromatic dispersion. The sensitivity of our device is remarkable and RCD with absolute value below $1 \mathrm{ps} / \mathrm{nm}$ can easily be detected. Our proof-ofprinciple experiments were based on a 10-GHz 2.4-ps signal, but additional numerical simulations have revealed the possibility to extend this concept to higher repetition rates and other pulse durations (using scaling laws [8], longer pulse durations can indeed enable a larger RCD measuring range up to several hundred $\mathrm{ps} / \mathrm{hm}$ ).

Based on a central filtering and anomalous dispersion regime, our experimental set-up has the advantage to require moderate input powers compared to other solutions reported so far. Moreover, the use of an anomalous segment of HNLF limits the detrimental pulse-to-pulse interactions and patterning effects [8] that may occur in normally dispersive fibers. Finally, from a practical implementation point of view, we believe that the compactness of our device could be greatly improved in a near future by replacing the fiber element by a highly nonlinear waveguides, for which constant progress in dispersion management now allows anomalous dispersion regime of propagation.

\section{REFERENCES}

[1] Z. Pan, et al., "Optical performance monitoring for the next generation optical communication networks," Opt. Fiber. Technol., vol. 16, pp. 20-45, 2010.

[2] P. Vorreau, et al., "Optical noise and dispersion monitoring with SOA-based optical 2R regenerator," IEEE Photon. Technol. Lett., vol. 17, pp. 244-246, 200.

[3] M. Pelusi, et al., "Multi-channel in-band OSNR monitoring using stimulated Brillouin scattering," Opt. Express, vol. 18, pp. 9435-9446, 4/2010 2010.

[4] R. Adams, et al., "All-optical in-band OSNR monitoring at $40 \mathrm{~Gb} / \mathrm{s}$ using a nonlinear optical loop mirror," IEEE Photon. Technol. Lett., vol. 18, pp. 469-471, 2006.

[5] T. T. Ng, et al., "In-band OSNR and chromatic dispersion monitoring using a fibre optical parametric amplifier," Opt. Express, vol. 13, pp. 5542-5552, 2005.

[6] T. D. Vo, et al., "Multi-impairment monitoring at $320 \mathrm{~Gb} / \mathrm{s}$ based on cross-phase modulation radiofrequency spectrum analyser," IEEE Photon. Technol. Lett., vol. 22, pp. 428-430, 3/2010 2010.

[7] P. S. Westbrook, et al., "Measurement of residual chromatic dispersion of a 40-Gb/s RZ signal via spectral broadening," IEEE Photon. Technol. Lett., vol. 14, pp. 346-348, 2002.

[8] J. Fatome and C. Finot, "Scaling guidelines of a soliton-based power limiter for 2R-optical regeneration applications," J. Lightw. Technol., vol. 28, pp. 2552-2556, 09/2010 2010.

[9] M. Asobe, et al., "Noise reduction of $20 \mathrm{Gbit} / \mathrm{s}$ pulse train using spectrally filtered optical solitons," Electron. Lett., vol. 34, pp. 1135-1136, 5/1998 1998.

[10] M. Gay, et al., "170 Gbit/s bit error rate assessment of regeneration using a saturable absorber and a nonlinear fiber based power limiter," IEEE Photon. Technol. Lett., vol. 22, pp. 158-160, 2/2010 2010.

[11] C. Finot and J. Fatome, "All-optical fiber-based ultrafast amplitude jitter magnifier," Opt. Express, vol. 18, pp. 18697-18702, 20102010.

[12] C. Finot and J. Fatome, "Experimental demonstration of an ultrafast all-optical bit-error indicating scheme," Microw. Opt. Technol. Lett., vol. 53, pp. 392-395, 2010. 